\title{
Влияние десорбции водорода на механические свойства и электронную структуру алмазоподобных углеродных нанониток
}

\author{
() А.И. Подливаев, Л.А. Опенов
}

Национальный исследовательский ядерный университет «МИФИ», 115409 Москва, Россия

E-mail: AlPodlivayev@mephi.ru

(Получена 11 октября 2016 г. Принята к печати 16 октября 2016 г.)

В рамках модели сильной связи изучено влияние десорбции водорода на механическую жесткость, спектр собственных колебаний и плотность электронных состояний углеродных нанониток с алмазоподобной структурой. Показано, что жесткость слабо меняется на начальной стадии десорбции и начинает заметно уменьшаться лишь после десорбции $\sim 20 \%$ атомов водорода. По мере десорбции в запрещенной зоне возникают энергетические уровни, которые в итоге формируют примесную зону.

DOI: $10.21883 /$ FTP.2017.05.44426.8423

\section{1. Введение}

Углеродные нанотрубки (УНТ) с хиральностью $(3,0)$ термодинамически неустойчивы: их энергии больше энергий соответствующих фрагментов графена [1]. После насыщения этих УНТ водородом до стехиометрии $\mathrm{H}: \mathrm{C}=1: 1$ они становятся устойчивыми, а в их электронной структуре появляется запрещенная зона [2] (будем использовать для таких полностью гидрированных УНТ обозначение $(3,0) \mathrm{H})$. Недавно открытые углеродные нанонитки („nanothreads“) [3] получаются из УНТ $(3,0) \mathrm{H}$ путем ряда трансформаций СтоунаУэльса (Stone-Wales, SW [4]) - поворотов связей C-C вместе с адсорбированными атомами водорода на угол $\sim 90^{\circ}$. В нанонитках протяженные области со структурой УНТ $(3,0) \mathrm{H}$ разделены компактными „дефектными“ участками с двумя трансформациями SW в каждом из них. Эти участки расположены по длине нанонитки случайным образом. Повернутые связи С-C „перегораживают“ полость УНТ, в результате чего формируются уникальные нанообъекты, отличные и от УНТ, и от обычных полимеров.

В нанонитках валентные орбитали всех атомов углерода $s p^{3}$-гибридизованы, как в алмазе (в отличие от УНТ с $s p^{2}$-гибридизацией углеродных орбиталей), а в расположении атомов углерода отчетливо прослеживается алмазоподобный тетраэдрический мотив. По этой причине нанонитки отличаются высокой прочностью и жесткостью [3,5], что сулит им разнообразные практические применения. Помимо своих выдающихся механических характеристик, нанонитки представляют интерес для приложений еще и как диэлектрические компоненты наноэлектронных приборов.

Основным каналом разрушения нанониток, особенно в устройствах с высокой рабочей температурой, является десорбция водорода, которая, как правило, предшествует развалу углеродного каркаса [6]. Цель настоящей работы заключается в расчете механической жесткости, спектров собственных колебаний и плотности электронных состояний нанониток с различным содержанием водорода. Мы показываем, что десорбция водорода приводит к уменьшению жесткости и к формированию примесной зоны, в которой находится уровень Ферми.

\section{2. Методы расчета}

В качестве модели нанонитки мы использовали фрагмент УНТ $(3,0) \mathrm{H}$ из 10 элементарных ячеек $\mathrm{C}_{12} \mathrm{H}_{12}$ [7] с одним дефектным участком (т.е. с двумя повернутыми на угол $\sim 90^{\circ}$ связями $\mathrm{C}-\mathrm{C}$ ) (см. рис. 1$)$. Граничные условия выбирались периодическими в направлении оси исходной УНТ (период определялся из требования минимальности энергии нанонитки) и свободными в двух поперечных направлениях.

Межатомные взаимодействия описывались в рамках неортогональной модели сильной связи [8], которая в явном виде учитывает квантово-механический вклад электронной подсистемы в полную энергию и достаточно хорошо описывает различные углеводородные наноструктуры (см. работы [6,7,9-11] и ссылки в них). Ранее мы использовали ее для моделирования графана [9], гидрированных нанотрубок [7], термической устойчивости нанониток [6] и пр.

Поскольку для квазиодномерных систем понятие площади поперечного сечения лишено смысла, мы определяли жесткость нанонитки $E$ как коэффициент пропорциональности между растягивающей силой $F$ и относительным удлинением $\varepsilon$, т.е. $E=F / \varepsilon$ (см. [5]). Мы ограничились малыми деформациями $\varepsilon \ll 1$ (линейный режим) и для определенности во всех расчетах полагали $\varepsilon=0.001$.

Спектры собственных колебаний нанониток рассчитывались путем численной диагонализации соответствующей матрицы вторых производных потенциальной энергии по координатам атомов с учетом различия масс водорода и углерода. Эти спектры затем использовались для определения плотности фононных состояний (phonon density of states, PDOS). 


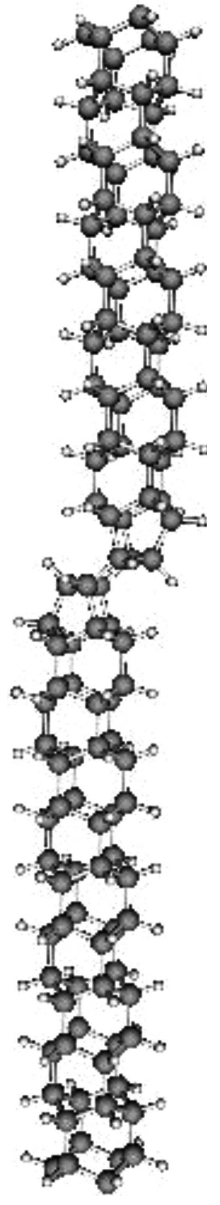

Рис. 1. Модельная нанонитка $\mathrm{C}_{120} \mathrm{H}_{120}$ с одним дефектным участком.

Компьютерное моделирование термической устойчивости нанониток [6] указывает на то, что в первом приближении вероятность десорбции того или иного атома водорода не зависит от его конкретного расположения (в области УНТ $(3,0)$ Н или в дефектном участке), т.е. примерно одинакова для всех атомов. По этой причине при каждой заданной стехиометрии Н:C мы удаляли из нанонитки соответствующее число атомов водорода случайным образом. При переходе от одного набора удаленных атомов к другому результаты существенно не изменяются, поэтому мы не выполняем усреднение по различным наборам, а приводим данные для одного из них (в разд. 3.2) или указываем интервал значений интересующей нас величины (в разд. 3.1). Помимо исходной бездефектной нанонитки $\mathrm{C}_{120} \mathrm{H}_{120}$, в настоящей работе мы рассматриваем нанонитки $\mathrm{C}_{120} \mathrm{H}_{119}, \mathrm{C}_{120} \mathrm{H}_{108}$, $\mathrm{C}_{120} \mathrm{H}_{96}, \mathrm{C}_{120} \mathrm{H}_{84}$ и $\mathrm{C}_{120} \mathrm{H}_{72}$, что отвечает десорбции одного атома, 10, 20, 30 и 40\% водорода соответственно.

\section{3. Результаты и их обсуждение}

В спектрах колебаний нанониток с различным содержанием водорода мнимые частоты отсутствуют, т.е. все рассмотренные нами атомные конфигурации являются метастабильными и отвечают локальным минимумам поверхности потенциальной энергии. PDOS нанонитки $\mathrm{C}_{120} \mathrm{H}_{120}$ (рис. 2) почти не отличается от PDOS соответствующей УНТ $(3,0) \mathrm{H}$ (различие не превышает $\sim 5 \%$ ). Максимумы PDOS при $f \sim 15,30$ и 35 ТГц обусловлены колебательными модами углеродного остова с различной поляризацией. Высокочастотный пик при $f \sim 95$ ТГц обязан своим происхождением вибрациям атомов водорода (см. также [12]). Десорбция из нанонитки 20\% водорода ведет к уменьшению максимумов PDOS при $f \approx 35$ и 95 ТГц на $\sim 30 \%$ (см. рис. 2). При последующей десорбции эти максимумы продолжают уменьшаться.

\section{1. Механическая жесткость}

Для жесткости бездефектной нанонитки $\mathrm{C}_{120} \mathrm{H}_{120}$ мы нашли $E=135$ нН. Полагая эффективный диаметр нанонитки равным $5 \AA$ [5], получим в привычных единицах $E \approx 700$ ГПа, что близко к модулю Юнга одностенных УНТ [13]. Десорбция одного атома водорода приводит к росту $E$ до 139-141нН. Причина такого (пусть и крайне незначительного) увеличения $E$ нам не вполне ясна. После десорбции 10 и 20\% водорода величина $E$ понижается до $131-132$ и 119-130 нН соответственно, что отчасти можно объяснить ослаблением ковалентных связей $\mathrm{C}-\mathrm{C}$ из-за уменьшения числа участвующих в их формировании электронов. При десорбции $30 \%$ водорода происходит разрыв 2-3 связей $\mathrm{C}-\mathrm{C}$. Вероятность их разрыва в области УНТ $(3,0) \mathrm{H}$ и в дефектном участке примерно одинакова. Жесткость таких нанониток составляет $E=105-129 \mathrm{HH}$, т.е. средняя величина $E$ уменьшается, а разброс значений $E$ существенно возрастает. Последнее обстоятельство свидетельствует о

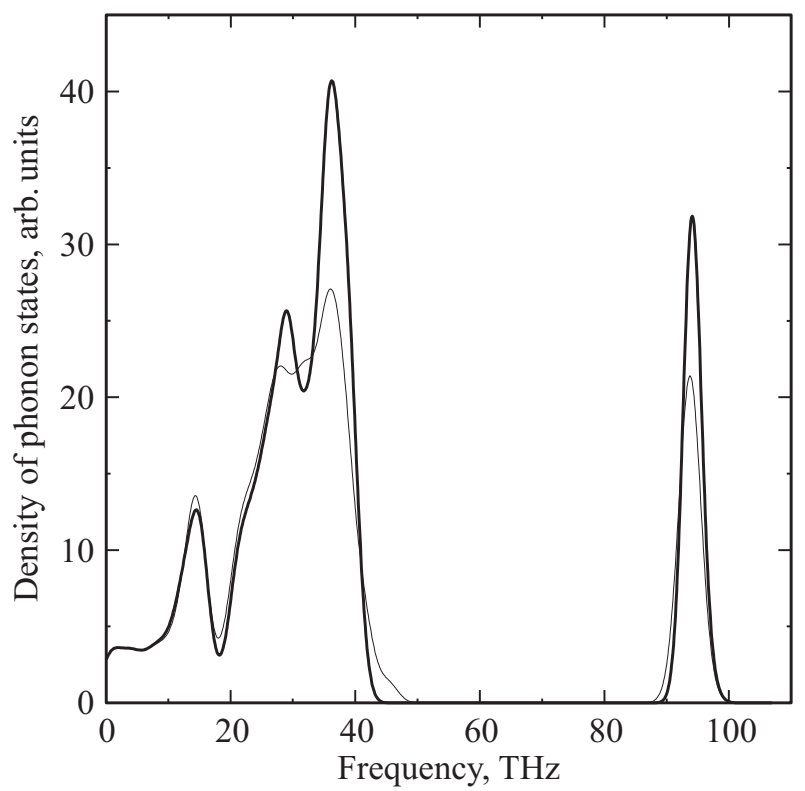

Рис. 2. Плотность фононных состояний нанонитки $\mathrm{C}_{120} \mathrm{H}_{120}$ до (жирная линия) и после (тонкая линия) десорбции $20 \%$ атомов водорода. 

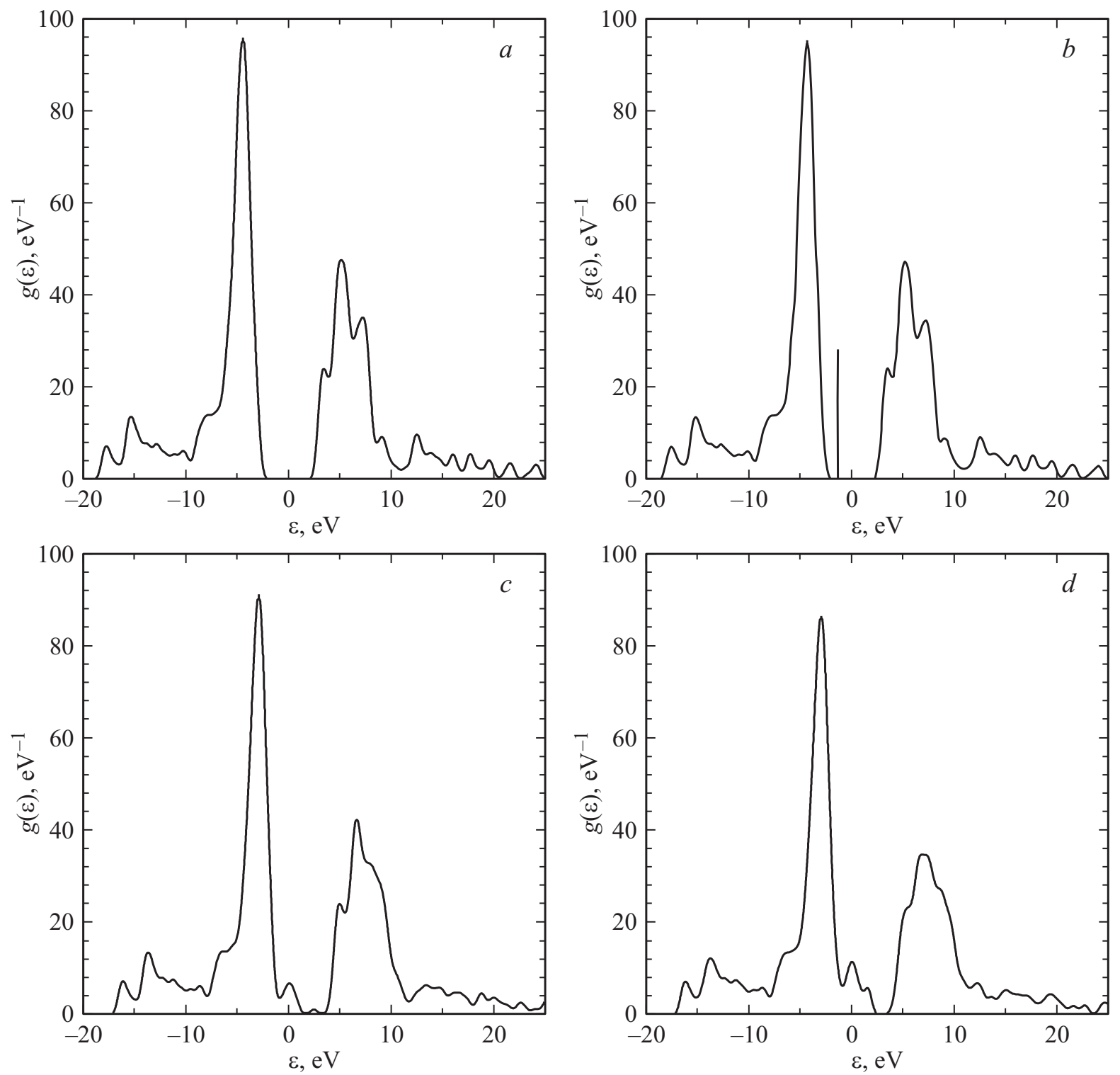

Рис. 3. Плотность электронных состояний $g(\varepsilon)$ в расчете на спин для нанониток $\mathrm{C}_{120} \mathrm{H}_{120}(a), \mathrm{C}_{120} \mathrm{H}_{119}(b), \mathrm{C}_{120} \mathrm{H}_{108}(c)$ и $\mathrm{C}_{120} \mathrm{H}_{96}(d)$. За начало отсчета энергии принят уровень Ферми.

сильной чувствительности $E$ к конкретному расположению водородных вакансий и разорванных связей $\mathrm{C}-\mathrm{C}$. После десорбции 40\% водорода жесткость варьирует в пределах 92-106 нН, продолжая уменьшаться.

Заметим, что полностью дегидрированные нанонитки термодинамически неустойчивы, поскольку представляют собой углеродные наноструктуры, энергия которых заведомо выше, чем у УНТ $(3,0)$ из-за трансформаций $\mathrm{SW}$, а УНТ $(3,0)$, в свою очередь, неустойчивы относительно трансформации в графеновые фрагменты [1]. Можно ожидать, что нанонитки с низким содержанием водорода тоже неустойчивы или как минимум близки к неустойчивости. По этой причине в настоящей работе мы не рассматриваем нанонитки, у которых содержание водорода составляет 50 и менее процентов от максимальной величины.

\section{2. Электронная структура}

Плотность электронных состояний нанонитки $\mathrm{C}_{120} \mathrm{H}_{120}$ приведена на рис. $3, a$. Видно, что эта нанонитка является диэлектрической. Ширина запрещенной зоны, определяемая как разность энергий нижней незаполненной (LUMO) и верхней заполненной (HOMO) молекулярной орбитали, составляет 6.0 эВ, как и в исходной УНТ $(3,0) \mathrm{H}$ [7]. Десорбция одного атома водорода приводит к появлению вблизи дна запрещенной зоны изолированного „Примесного“ уровня энергии (рис. $3, b)$. По мере десорбции все новых и новых атомов водорода число таких уровней увеличивается. Таким образом, на начальной стадии десорбции нанонитки по своим электрическим характеристикам аналогичны слабо легированным полупроводникам. После десорбции 10\% водорода фор- 
мируется примесная зона (рис. 3, c). При дальнейшей десорбции положение этой зоны внутри диэлектрической щели и плотность электронных состояний в ней изменяются нерегулярно. Примечательно, однако, что уровень Ферми всегда находится в примесной зоне, а плотность состояний на уровне Ферми отлична от нуля (рис. $3, c, d)$. В силу этого частично дегидрированные нанонитки при низких температурах должны быть проводящими.

\section{4. Заключение}

1. При десорбции из нанониток менее $10 \%$ водорода их механическая жесткость почти не изменяется. После десорбции 20-30\% водорода жесткость уменьшается на $\sim 30 \%$.

2. По мере десорбции водорода внутри диэлектрической щели формируется примесная зона, в которой находится уровень Ферми, что может приводить к металлизации исходно непроводящих нанониток.

3. Десорбция $20 \%$ водорода ведет к уменьшению максимумов плотности фононных состояний при 35 и 95 ТГц на 30\%.

Работа выполнена в НИЯУ МИФИ при финансовой поддержке Российского научного фонда (проект 14-22-00098).

\section{Список литературы}

[1] L.-M. Peng, Z.L. Zhang, Z.Q. Xue, Q.D. Wu, Z.N. Gu, D.G. Pettifor. Phys. Rev. Lett., 85, 3249 (2000).

[2] D. Stojkovic, P. Zhang, V.H. Crespi. Phys. Rev. Lett., 87, 125502 (2001).

[3] T.C. Fitzgibbons, M. Guthrie, E. Xu, V.H. Crespi, S.K. Davidowski, G.D. Cody, N. Alem, J.V. Badding. Nature Mater., 14, 43 (2015).

[4] A.J. Stone, D.J. Wales. Chem. Phys. Lett., 128, 501 (1986).

[5] R.E. Roman, K. Kwan, S.W. Cranford. Nano Lett., 15, 1585 (2015).

[6] Л.А. Опенов, А.И. Подливаев. Письма ЖЭТФ, 104, 192 (2016).

[7] А.И. Подливаев, Л.А. Опенов. ФТП, 51, 222 (2017).

[8] M.M. Maslov, A.I. Podlivaev, K.P. Katin. Molecular Simulation, 42, 305 (2016).

[9] Л.А. Опенов, А.И. Подливаев. Письма ЖТФ, 36, 69 (2010).

[10] А.И. Подливаев, Л.А. Опенов. ФТП, 45, 988 (2011).

[11] Л.А. Опенов, А.И. Подливаев. ФТП, 46, 210 (2012).

[12] H. Zhan, G. Zhang, Y. Zhang, V.B.C. Tan, J.M. Bell, Y. Gu. Carbon, 98, 232 (2016)

[13] M.M.J. Treacy, T.W. Ebbesen, J.M. Gibson. Nature, 381, 678 (1996).

Редактор Г.А. Оганесян

\section{Effect of hydrogen desorption on mechanical characteristics and electronic structure of diamond-like carbon nanothreads}

\author{
A.I. Podlivaev, L.A. Openov \\ National Research Nuclear University «MEPhl», \\ 115409 Moscow, Russia
}

\begin{abstract}
Effects of hydrogen desorption on mechanical stiffness, vibrational spectra, and electron density of states of carbon nanothreads with diamond-like structure are studied within the framework of tight-binding model. It is shown that stiffness changes little at the initial stage of desorption and starts to decrease significantly only after desorption of $\sim 20 \%$ of hydrogen atoms. Upon desorption, energy levels appear within the bandgap which finally form the impurity band.

